\title{
Parameter Sensitivity in Subsurface Gas Transport Calculations
}

\author{
TODD R. ZEITLER*, KRISTOPHER KUHLMAN, JEFFERY \\ GREATHOUSE
}

Sandia National Laboratories, Albuquerque, NM 87185, USA (*correspondence: tzeitle@sandia.gov, klkuhlm@sandia.gov, jagreat@sandia.gov)

To better understand the impacts of host rock properties on subsurface gas transport, a multi-scale modeling and laboratory experiment approach has been undertaken. Coordinated adsorption experiments, molecular modeling, and transport modeling will allow us to better understand the effect of chemical and physical interactions at the gas-surface interface with the goal of better predicting flow and transport in porous media. The work presented here focuses on the results of transport calculations with an emphasis on the sensitivity of transport times to model input. A framework has been developed that allows for later incorporation of experimental and molecular-scale modeling results.

Previous studies have found that gases are transported to the surface mostly by flow along faults and fractures driven by barometric pressure variations.[1] Diffusive transport in the surrounding matrix also plays an important role.[2]

In this work, radionuclide gas transport in a variety of subsurface settings (natural rock/mineral phases with a variety of pore size distributions, fractured/damaged phases) is investigated to look at the transport mechanisms that drive gas migration in the subsurface toward the Earth's surface.

The PFLOTRAN subsurface flow and reactive transport code [3] has been used to perform an array of subsurface gas transport calculations over $1 \mathrm{D}$ and $2 \mathrm{D}$ domains. The primary input variables of pressure, temperature, porosity, permeability, diffusion coefficient, and matrix partition coefficient were varied over expected ranges to obtain transport time over a fixed domain. Results also include gas concentration (saturation) profiles as a function of time, including cumulative amounts reaching the ground surface for each gas component (i.e., "breakthrough").

Finally, a parameter sensitivity analysis was used in order to provide insight into the relative importance of the uncertain input variables to the calculated transport times.

SNL is managed and operated by NTESS under DOE NNSA contract DE-NA0003525

[1] C. R. Carrigan, et al. (1996). Nature, 382, 528-531. [2] M. J. Paul, et al. (2018). J. Environ. Radioact., 187, 65-72. [3] Hammond, G. E., et al. (2014). Water Resources Research 50(1): 208-228. 